\title{
671 BIOLOGICAL IMPACTS OF STANDARD OF CARE CHEMOTHERAPIES ON IMMUNE EFFECTOR CELLS FROM AML PATIENTS
}

${ }^{1}$ Dmitry Zhigarev*, 'Alexander MacFarlane, ${ }^{2}$ Christina Drenberg, ${ }^{1}$ Reza Nejati, ${ }^{1}$ Asya Varshavsky, ${ }^{1}$ Kerry Campbell. ${ }^{1}$ Fox Chase Cancer Center, Philadelphia, PA, United States; ${ }^{2}$ Janssen $R \& D$, Spring House, PA, United States

Background Acute myeloid leukemia (AML) is a heterogeneous group of malignant bone marrow diseases, characterized by massive and uncontrolled proliferation of myeloid precursor cells, which alters normal blood cell ratios. This disease is common to older adults and collectively displays one of the lowest 5 -year overall survival rates $(<25 \%)$ among all cancers, currently representing the deadliest form of leukemia. Improved treatments are clearly needed, and immunotherapies are attractive candidate therapies to explore.There are currently several standard chemotherapeutic treatment schemes for AML, which could be divided into two major groups: (1) cytotoxic chemotherapy (" $7+3$ " or daunorubicin-cytarabine) and (2) hypomethylating agents (HMAs). HMAs include both 5 -azacytidine and decitabine, which are cytidine analogs that inhibit DNA methyltransferase, resulting in the hypomethylation of DNA and inducing expression of silenced gene loci. Currently, HMAs are routinely delivered in combination with the Bcl-2 inhibitor venetoclax.The goals of this study are to determine how these standard first line therapies can affect the frequency and functional integrity of effector immune cells in patients' blood and establish when the phenotype and function of immune cells are restored to identify time windows when second line immunotherapies could be most effective.

Methods More than 100 blood samples were obtained from 33 previously untreated AML patients. More than 50 measurable biomarkers were analyzed using 14-color flow cytometry to assess immune phenotypes of $\mathrm{T}$ and $\mathrm{NK}$ cells in peripheral blood of AML patients prior to treatment and at up to four timepoints after initiation of treatment with HMA or chemotherapy.

Results We found several significant changes in immune cell phenotype and function that occur in response to these therapies. Treatment with HMAs was strikingly less impactful on immune cells in patients compared to previously published in vitro studies. Nevertheless, HMA treatment increased perforin levels in $\mathrm{T}$ and $\mathrm{NK}$ cells, inhibited IFN-gamma secretion by $\mathrm{CD} 8+\mathrm{T}$ cells, and changed expression of several checkpoint molecules. While chemotherapy caused fewer phenotypic changes it dramatically decreased the total number of immune cells. We also determined viable, functional and phenotypical recovery periods for immune effector cells after the treatments.

Conclusions Our results are important for introducing new second line immunotherapies to these chemotherapeutic regimens for treating AML and to improve overall understanding of immune cell behavior under conditions of anti-tumor treatment.

Acknowledgements Supported by grants from Janssen and the U.S./Israel Binational Science Foundation.

Ethics Approval The study was approved by the Fox Chase Cancer Center Institutional Review Board, approval number 17-8010, and all patients provided informed consent before taking part in the study.

http://dx.doi.org/10.1136/jitc-2021-SITC2021.671 